Artículos científicos

\title{
Intervención organizacional por competencias profesionales en una empresa de proveeduría de calzado dentro del estado de Guanajuato
}

Organizational intervention for professional skills in a shoe supply company in the State of Guanajuato

Intervenção organizacional para qualificação profissional em empresa fornecedora de calçados do estado de Guanajuato

Adriana Fragoso Mora

Tecnológico Nacional de México, Instituto Tecnológico Superior de Purísima del Rincón, México adriana.fm@purisima.tecnm.mx https://orcid.org/0000-0001-6434-8485

Laura Marisela Martínez Sánchez

Tecnológico Nacional de México, Instituto Tecnológico Superior de Purísima del Rincón, México laura.ms@purisima.tecnm.mx https://orcid.org/0000-0002-1112-1700

Alba Edith Elías Chávez

Tecnológico Nacional de México, Instituto Tecnológico Superior de Purísima del Rincón, México alba.ec@purisima.tecnm.mx https://orcid.org/0000-0001-8325-7095 


\section{Resumen}

El objetivo de esta investigación fue determinar el impacto de las competencias blandas de personas que ocupan puestos gerenciales en una empresa de calzado guanajuatense con el fin de respaldar el modelo de gestión tecnológica. Para ello se empleó una metodología de caso, la cual fue desarrollada siguiendo cuatro fases: fundamentos y diseño de instrumentos, selección de puestos tipo y aplicación de instrumentos, validación por expertos, y evaluación al desempeño piloto. Algunos beneficios detectados fueron los siguientes: diagnosticar situacionalmente a la organización, identificar las necesidades de capacitación y evaluar mejor el desempeño traducido en el comportamiento de los gerentes, implementar un sistema de evaluación capaz de neutralizar la subjetividad, y proponer medidas orientadas a mejorar el comportamiento y la comunicación. La empresa objeto de estudio, en síntesis, logró determinar cuáles colaboradores requieren de un proceso de mejora continua, así como seleccionar a los que tienen condiciones de promoción para estimular la productividad y mejorar las relaciones humanas en el trabajo.

Palabras clave: descripción del empleo, evaluación de personal, gestión de competencias, organización.

\section{Abstract}

The research of this research was to determine the impact of the soft skills of people who occupy managerial positions in Guanajuato Footwear Company in order to support the technology management model. For this, a case methodology was used, which was developed following four phases: fundamentals and design of instruments, selection of standard positions and application of instruments, validation by experts, and evaluation of pilot performance. Some benefits detected were the following: situationally diagnosing the organization, identifying training needs and better evaluating performance translated into the behavior of managers, implementing an evaluation system capable of neutralizing subjectivity, and proposing measures aimed at improving behavior and communication. The company under study, in short, sought to select employees, as well as select a continuous improvement process, as well as select those who have promotional conditions to stimulate productivity and improve human relations at work.

Keywords: job description, staff evaluation, competendy management, organization. 


\section{Resumo}

O objetivo desta pesquisa foi determinar o impacto das habilidades sociais de pessoas que ocupam cargos gerenciais em uma empresa calçadista de Guanajuato para apoiar o modelo de gestão tecnológica. Para isso, foi utilizada uma metodologia de caso, que foi desenvolvida seguindo quatro fases: fundamentos e desenho dos instrumentos, seleção das posições padrão e aplicação dos instrumentos, validação por especialistas e avaliação do desempenho do piloto. Alguns benefícios detectados foram os seguintes: diagnosticar situacionalmente a organização, identificar necessidades de treinamento e avaliar melhor o desempenho traduzido no comportamento dos gestores, implantar um sistema de avaliação capaz de neutralizar a subjetividade e propor medidas que visem melhorar o comportamento e comunicação. A empresa em estudo, em suma, conseguiu determinar quais são os colaboradores que requerem um processo de melhoria contínua, bem como selecionar aqueles que têm condições de promoção para estimular a produtividade e melhorar as relações humanas no trabalho.

Palavras-chave: descrição do trabalho, avaliação de pessoal, gestão de competências, organização.

Fecha Recepción: Mayo 2020

Fecha Aceptación: Diciembre 2020

\section{Introducción}

El papel que desempeñan las empresas en el mercado es de gran relevancia para un correcto y eficaz funcionamiento de la economía de los países. Por eso, Palomo y Pedroza (2018) señalan que "administrar de manera integral y efectiva la empresa, incluyendo las ventas, la calidad, la entrega, el mercado, la rotación de personal y la rentabilidad del negocio van de la mano con la tecnología y el aprovechamiento eficiente de sus recursos" (párr. 07). Una empresa comprometida con la investigación, el desarrollo y la innovación requiere de talento humano capacitado para crear y ejecutar proyectos, de ahí la importancia de detectar y formar esas habilidades en el personal para que las organizaciones puedan atender las exigencias del mercado.

El objetivo de esta investigación, por tanto, fue determinar el impacto de las competencias blandas de personas que ocupan puestos gerenciales en una empresa de proveeduría de calzado guanajuatense con el fin de respaldar su modelo de gestión tecnológica. 


\section{Revisión de la literatura}

La competitividad de las empresas ha sido un tema estudiado por varios autores. Por ejemplo: (Müller, 1995) la propone como la «capacidad sostenida para ganar y mantener una participación lucrativa en el mercado «relacionan dicho término con la obtención de una ventaja basada en la generación de productos o procesos superiores para lograr una mejor posición en el mercado. Es decir, incorpora el objetivo de logro industrial"

Desde la obra de Müller: el Caleidoscopio de la Competitividad; "Competitividad es la habilidad sostenible de obtener ganancias y mantener la participación en el mercado (...) Esta definición presenta tres importantes y mensurables dimensiones: ganancias; participación en el mercado, y, a través de la palabra 'sostenibilidad', registra el aspecto temporal" (Duren, Martin y Westgren, 1992, p. 2).

En este sentido, cabe destacar que la percepción sobre el papel que juega la pequeña empresa en el desarrollo de un país está cambiando, puesto que ahora no se considera vigente la idea de una estructura económica dual formada por un núcleo de empresas que ofrecen empleos permanentes y que se encuentran rodeadas por un numeroso grupo de pequeñas y volátiles empresas que actúan de manera subsidiaria.

Por tanto se acepta que la ventaja competitiva se genera a nivel de empresa y de industrias específicas. La percepción anterior, "construye la base de un alto nivel de consenso sobre el hecho de que el complejo de políticas públicas y de relaciones entre las empresas e instituciones que rodean a cada industria conforma el ambiente competitivo, lo que Porter (1990) llama el diamante de la ventaja nacional" (Solleiro \& Castañón, 2005, p. 3).

Siguiendo esta idea, se considera que la presente investigación constituye un medio para generar habilidades de evaluación con trabajo de aplicación y diseño por competencias, labor que no se debe asociar con el dictamen de pasos a seguir tipo formulario o receta (Cazáres y Cuevas de la Garza, 2007). Esto se debe a que el método empleado es flexible, de modo que cada quien lo puede ajustar a sus necesidades

Lógicamente, la búsqueda de la diferenciación competitiva sostenible y la estructura organizacional para mantener la eficiencia de la empresa se puede efectuar mediante el desarrollo de una cualidad distintiva, la cual debe ser inimitable en el corto plazo para que ofrezca beneficios. Esto se puede impulsar con apoyo en las capacidades tecnológicas, las cuales deben ser consideradas como una facultad genérica de la empresa para movilizar recursos tecno-científicos individuales. Estos recursos permiten la mejora o creación de nuevos productos innovadores de éxito, y están al servicio de la 
implantación de estrategias competitivas eficaces para neutralizar las amenazas y explotar las oportunidades que ofrece el entorno. En otras palabras, las capacidades tecnológicas no solo demandan la aplicación del conocimiento existente o la adquisición de uno nuevo, sino también la diferenciación del conocimiento en el que se sustentan (García y Navas, 2007; Levinthal y March, 1993).

\section{Planteamiento del problema}

La Organización para la Cooperación y Desarrollo Económico OECD (1992) concluye que los factores que influyen en la competitividad al nivel de la empresa incluyen:

El manejo exitoso de los flujos de producción, materias primas e inventarios.

La gestión exitosa de mecanismos de interacción entre planeación mercadotécnica, I+D formal, diseño, ingeniería y producción industrial.

La capacidad de combinar actividades internas de I+D e innovación con la cooperación tecnológica con universidades y otras empresas.

La capacidad de incorporar definiciones más exactas de las características de la demanda y de la evolución de los mercados en estrategias de diseño y producción.

La capacidad de organizar relaciones interempresariales exitosas con proveedores de materiales y componentes y clientes.

Los pasos seguidos para mejorar las capacidades de trabajadores y empleados a través de inversiones en entrenamiento especializado, así como en la generación de niveles más altos de responsabilidad del trabajador en la producción. (Solleiro \& Castañón, 2005, p. 6-7).

No obstante, México suele ocupar las últimas posiciones en cuanto a desarrollo de competitividad refiere, lo cual puede ser la consecuencia de una escasa innovación que no se promueve desde una integración entre la universidad, la industria y el gobierno (modelo de la triple hélice) (Leydesdorff y Etzkowitz, 1996). La muestra de ello es un marco legal deficiente y las limitaciones de gobernabilidad en el sistema de innovación. Aun así, actualmente en el país se realizan esfuerzos para superar esas debilidades a través, por ejemplo, de la comercialización de la investigación pública y la creación de condiciones para el emprendimiento innovador.

En el caso concreto de este trabajo, el enfoque se ha puesto en el sector del calzado, el cual se encuentra constituido por 7981 empresas divididas en grandes, 
medianas, pequeñas y micros (figura 1) que distribuyen a nivel nacional unos 254 millones de pares de piezas al año (figura 2):

Figura 1. Tamaño de empresas del sector calzado

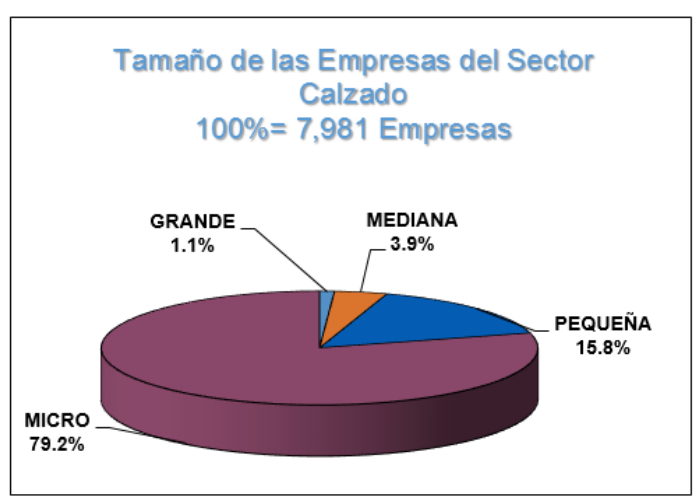

Fuente: Elaboración propia de la empresa con base en datos Instituto Nacional de Estadística Geografía e Informática-INEGI-Mexico (2007-2016).

Figura 2. Distribución de la producción de calzado por tamaño de empresa

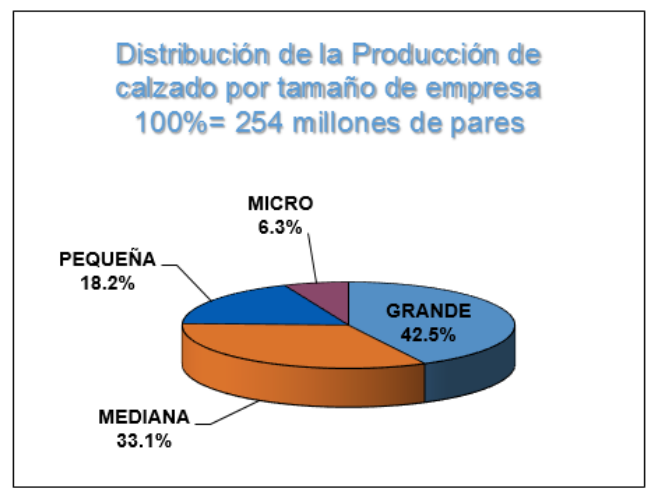

Fuente: Elaboración propia de la empresa con base en datos Instituto Nacional de Estadística Geografía e Informática-INEGI-Mexico (2007-2016).

La evolución de la producción del sector calzado se muestra en la figura 3, donde se ofrece un comparativo entre los años 2001 y 2016. 
Figura 3. Evolución de la producción de calzado por zona geográfica 2001-2016

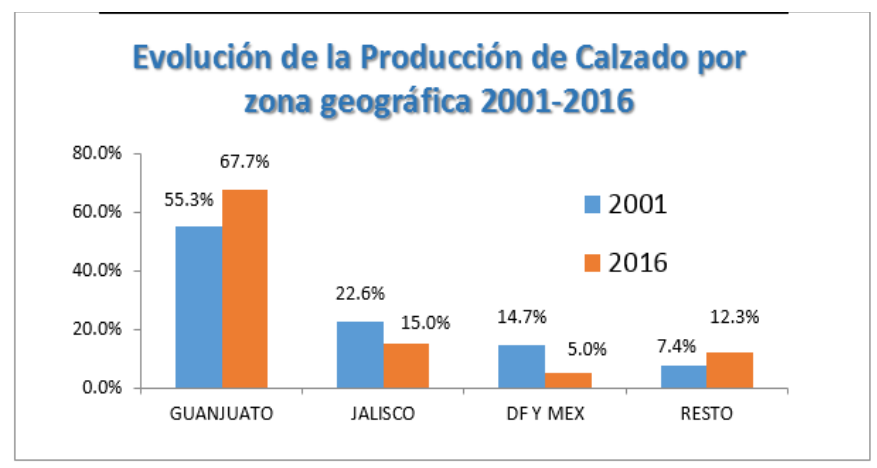

Fuente: Elaboración propia de la empresa con base en datos Instituto Nacional de Estadística Geografía e Informática-INEGI-Mexico (2007-2016).

Ahora bien, la organización objeto de estudio del presente trabajo fue constituida el 27 de octubre de 1978, aunque inició sus operaciones en 1980 con la finalidad de cubrir la necesidad de brindar el suministro de hormas de plástico a la industria del calzado, para lo cual contó con la asesoría de empresas italianas. Actualmente, tiene aproximadamente 102 colaboradores, y sus principales productos son los siguientes: impresión 3D, horma de cuarta generación y horma de placa integrada o quinta generación, los cuales son comercializados dentro del país, así como en otras naciones (p. ej., Canadá, Estados Unidos, Guatemala, El Salvador, Chile, Perú, Colombia, República Dominicana).

Desde sus inicios la organización ha estado comprometida con la investigación y desarrollo de la innovación, lo cual se evidencia en su trayectoria de 40 años de permanencia en los distintos mercados nacionales e internacionales. En 2017 fue galardonada con el Premio Nacional de Tecnología e Innovación, ya que fue creadora de su modelo de gestión tecnológica. A pesar de estos logros, sin embargo, la empresa también percibió diversos problemas relacionados con factores de índole actitudinal y gerencial por parte del personal gerencial, así como de los mandos medios y operativos. En otras palabras, se detectaron constantes errores y falta de especificaciones claras con los clientes, lo cual dificultaba la resolución de problemas.

\section{Objetivo general}

Identificar las competencias profesionales actitudinales del personal gerencial y de mandos medios de la organización con la finalidad de evaluar su desempeño actual y su impacto en la implementación del modelo de gestión tecnológica en sus áreas productivas para el logro de resultados esperados hacia sus clientes y hacia la dirección de la empresa. 


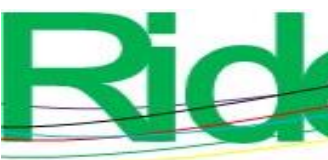

Revista Iberoamericana para la Investigación y el Desarrollo Educativo ISSN $2007-7467$

Vale precisar que el término capacidad o competencia profesional se refiere a las actuaciones integrales concretadas para identificar, interpretar, argumentar y resolver problemas del contexto de la forma más ética e idónea posible, articulando el saber ser, saber hacer y saber conocer (Tobón, 2012). En socio-formación, es clave servir a los demás, al ambiente y a sí mismo para poder lograr la formación integral y el desarrollo de las competencias necesarias. Esto se hace contribuyendo a resolver necesidades y problemas concretos con base en estrategias tales como el aprendizaje basado en problemas y la ejecución de proyectos. "No basta tener saberes, hay que aplicarlos en la transformación del mundo" (Tobón, 2012, p. 4).

\section{Justificación}

Esta investigación de tipo explicativa, descriptiva y correctiva se justifica porque pretende aportar conocimiento en el ámbito de las competencias del saber, el hacer y el saber transferir a través de la propuesta de diagnóstico de necesidades de capacitación y evaluación al desempeño gerencial bajo la metodología 360 grados. Con esto se procura transformar la experiencia en la gestión en el desempeño gerencial como aprendizaje significativo y organizacional hacia el interior de la empresa.

\section{Hipótesis}

Al identificar las competencias profesionales actitudinales del personal gerencial, así como de los mandos medios de la organización se puede determinar que existe diferencia significativa en la implementación del modelo de gestión tecnológica en sus áreas productivas para el logro de resultados esperados hacia sus clientes $\mathrm{y}$, en consecuencia, hacia la dirección de la empresa.

\section{Metodología}

\section{Desarrollo del estudio}

En la investigación se empleó una metodología de caso, la cual fue desarrollada en las siguientes fases: 


\section{Fase 1: Fundamentos y diseño de instrumentos}

Se llevó a cabo una reunión inicial con la gerencia general de la empresa de calzado para acordar el plan de intervención. En este primer encuentro se escucharon las necesidades para determinar las competencias necesarias según el análisis del modelo de gestión tecnológica de la empresa, así como la funcionalidad y el desarrollo de cada una de sus funciones estratégicas: vigilar, planear, habilitar, proteger e implementar (figura $4)$.

Figura 4. Fase 1: Fundamentación y diseño de instrumentos
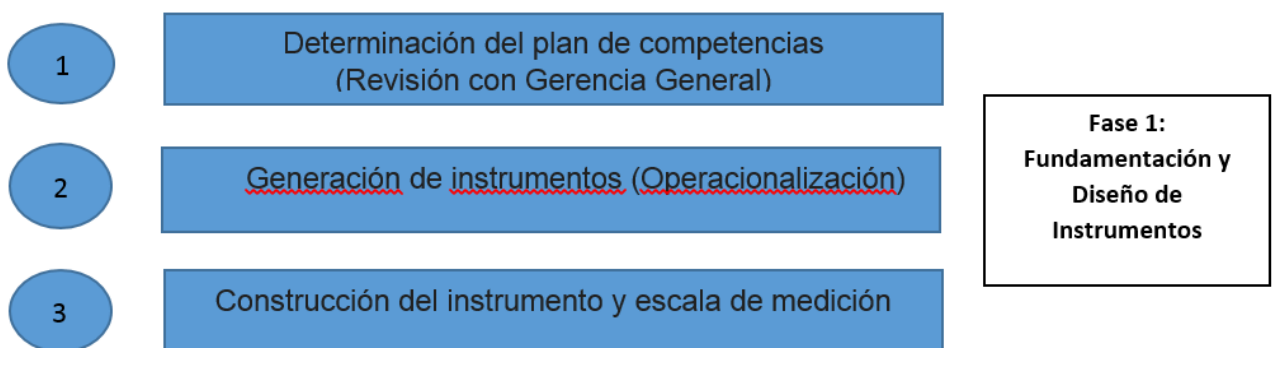

Fuente: Elaboración propia

Por otra parte, se concretó con los gerentes la sesión de trabajo en el taller, donde se les facilitó un formato para que describieran el alcance de sus responsabilidades traducidas en competencias profesionales. Para ello, se acordaron las siguientes escalas de medición:

- Nivel 1: Competencia no necesaria

- Nivel 2: Competencia baja

- Nivel 3: Competencia intermedia

- Nivel 4: Competencia en dominio alto y profundo

- Nivel 5: Competencia en grado experto y con capacidad para enseñar a otros.

Igualmente, se analizó el alcance por posición de la responsabilidad según las competencias genéricas y las técnicas requeridas para la entrega de resultados acordes al modelo de gestión tecnológica.

\section{Fase 2: Selección de puestos tipo y aplicación de instrumentos}

El entregable del taller gerencial fue el descriptivo de puestos desarrollado por cada gerente. Luego, cada uno de ellos fue entrevistado para concretar el alcance, las competencias genéricas y las necesarias para el modelo de gestión tecnológica. Asimismo, se implementó el instrumento para detectar las necesidades de capacitación (DNC) de los ocupantes de los puestos respecto a las competencias determinadas en las 
descripciones de puesto. Se procesaron los datos de forma estadística de acuerdo con los niveles de competencia requeridos y las prioridades de capacitación a cubrir según las competencias genéricas y de gestión tecnológica para determinar de forma gráfica las brechas de capacitación a cubrir para la elaboración del plan anual de capacitación (figura $5)$.

Figura 5. Fase 2: Aplicación de instrumentos y análisis gráfico

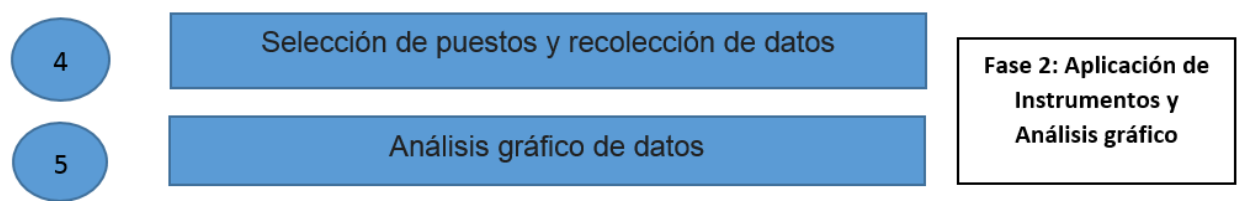

Fuente: Elaboración propia

\section{Fase 3: Validación por expertos}

De acuerdo con Rodríguez (2005), Arias (2000), Pérez (2000), entre otros, la triangulación de datos puede ser orientada en el tiempo y en el espacio, de persona, de investigadores, de teorías, de métodos o múltiple. En la presente investigación se utilizó la triangulación de datos. El objetivo fue verificar las tendencias detectadas en un determinado grupo de observaciones.

La confrontación de los datos puede estar basada en criterios espacio-temporales y niveles de análisis. Según Arias (2000), en esta tipología se considera como el uso de múltiples fuentes de datos para obtener diversas visiones acerca de un tópico para el propósito de validación. A su vez, la triangulación de datos en el tiempo implica validar una proposición teórica relativa a un fenómeno en distintos momentos.

Dentro de la presente investigación, la triangulación se realizó por persona, es decir, es la recolección de datos del diagnóstico de necesidades de capacitación (DNC) y su correspondiente evaluación al desempeño (metodología 360 grados) en al menos dos de los tres niveles de persona: individuos (gerentes), grupos o colectivos (áreas o departamentos). La recolección de datos de una fuente se utilizó para validar los datos de las otras fuentes o de una sola. Cada nivel de datos fue contrastado para validar los hallazgos del otro nivel (figura 6). 
Figura 6. Fase 3: Validación por expertos

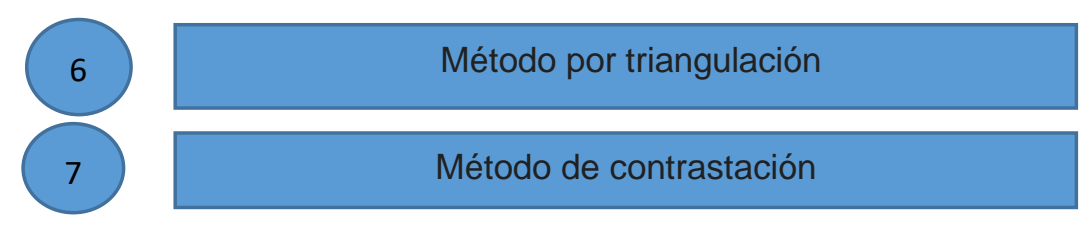

Fuente: Elaboración propia

\section{Fase 4: Evaluación al desempeño piloto (recolección de información)}

Se estableció el perfil de puesto a partir de las competencias blandas identificadas como necesarias para cada posición gerencial en las fases 1,2 y 3 del proyecto; en este sentido, se organizó el proceso de evaluación 360 grados en forma íntegra con los gerentes, incluyendo una autoevaluación, evaluación de su jefe inmediato y de un compañero par al respecto. Con esto de forma gráfica se obtuvieron los resultados para su análisis, discusión colaborativa entre evaluadores (personal del ITSPR), evaluados (gerentes) y director general para su mayor comprensión del proceso de evaluación y la retroalimentación grupal para su propia mejora continua (figura 7).

Figura 7. Fase 4: Evaluación al desempeño

\section{8}

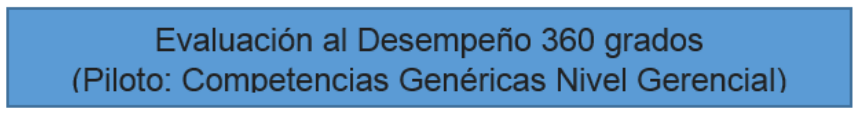

Fase 4: Evaluación al Desempeño

Fuente: Elaboración propia

La evaluación del desempeño por el método de 360 grados en una empresa mexicana indicó que la ED está relacionada con establecer objetivos y estándares de desempeño, que permiten medir el ejercicio contra dichos estándares y brindar retroalimentación a los empleados (Sapien-Aguilar, Gutiérrez Diez, Piñón Howlet y Araiza Zapata, 2016).

Dado lo anterior, el desarrollo de la fase 4 en la presente investigación consistió en evaluar el desempeño utilizando el método 360 grados con base en tres grupos de competencias: genéricas, gerenciales para respaldar su modelo de gestión tecnológica y técnicas específicas. Este conocimiento permitirá mejorar la interacción entre los empleados y la empresa para el logro de un beneficio en común, posibilitando la creación de las condiciones para un mejor desempeño. El método de 360 grados es una herramienta de gestión del talento humano que pretende — a partir de los objetivos organizacionales- 


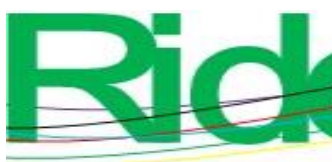

Revista Iberoamericana para la Investigación y el Desarrollo Educativo ISSN $2007-7467$

valorar las conductas y actitudes que definen el modo en que cada persona está llevando a cabo su tarea.

\section{Resultados}

El proceso de diagnóstico y evaluación de las competencias gerenciales blandas son relevantes por el grado de desarrollo que requieren los colaboradores de la organización objeto de estudio; en este sentido se identificaron las siguientes: liderazgo situacional, inteligencia emocional, capacidad de escuchar a otros, negociación, sentido de innovación, trabajo en equipo, iniciativa, gestión del talento humano, habilidad para comunicar, administración de prioridades y tolerancia.

La estrategia competitiva de la organización está basada en la innovación de productos, procesos y servicios. De hecho, desde hace siete años se ha tenido en mente la diferenciación de productos y servicios, de ahí que se haya realizado la inversión en I+D con el fin de satisfacer las necesidades de sus clientes y con ello obtener mejores resultados. El tipo de venta que se realiza es directa; sin embargo, para atender mejor a los clientes se ha creado una estrategia de marketing digital utilizando el internet y las redes sociales.

Como parte de esta estrategia competitiva se ha trabajado en la identificación de los competidores nacionales y extranjeros. Es importante mencionar que la organización está en un nicho de mercado premium, es decir, atiende a clientes y marcas reconocidas, por la alta tecnificación utilizada en la producción de las hormas, así como el personal altamente calificado, por lo que no es posible competir con hormeros de una tecnificación menor.

Las principales ventajas competitivas de sus productos son la alta precisión dimensional y el acabado automático de la punta y del talón de las hormas, que son las áreas críticas en el biodimensionamiento de la anatomía de la horma, dando un calce perfecto para la manufactura del calzado sin la intervención de la mano de obra.

En cuanto a la más destacable ventaja comparativa que ha logrado obtener la organización con los proyectos de innovación tecnológica implementados en los últimos tres años (lo que constituye un factor clave del éxito en el mercado), se puede mencionar la velocidad de respuesta en el diseño y desarrollo de prototipos de hormas para calzado, con la más alta calidad y precisión dimensional. Hoy los fabricantes de calzado reconocen que la clave para competir globalmente se encuentra en la velocidad para introducir un 
nuevo producto al mercado (speed to market) sin sacrificar calidad (Premio Nacional de Tecnología e Innovación PNTi, 2017).

Sin duda, esta es un organización comprometida con la investigación, el desarrollo tecnológico y la innovación (I+D+i). Por eso, se invierte $10 \%$ de las ventas mensuales para realizar pruebas y buscar nuevos métodos o sistemas de producción; gracias a ello se ha logrado la reducción de desperdicios y pérdidas, lo cual ha aumentado hasta en 50 $\%$ la utilidad y el valor de las acciones.

Sobre el diagnóstico situacional en la organización, se determinaron las competencias genéricas relevantes para respaldar el modelo de gestión tecnológica de la empresa: pensamiento sistémico, vigilancia tecnológica, normatividad en propiedad industrial, investigación, gestión de nuevas tecnologías, proyectos en gestión tecnológica, innovación del conocimiento, entre otras, cuantificando enseguida la evaluación que se obtuvo al respecto en cada función del modelo de gestión estratégica de la empresa (figura 8).

Figura 8. Modelo de gestión tecnológica de la empresa

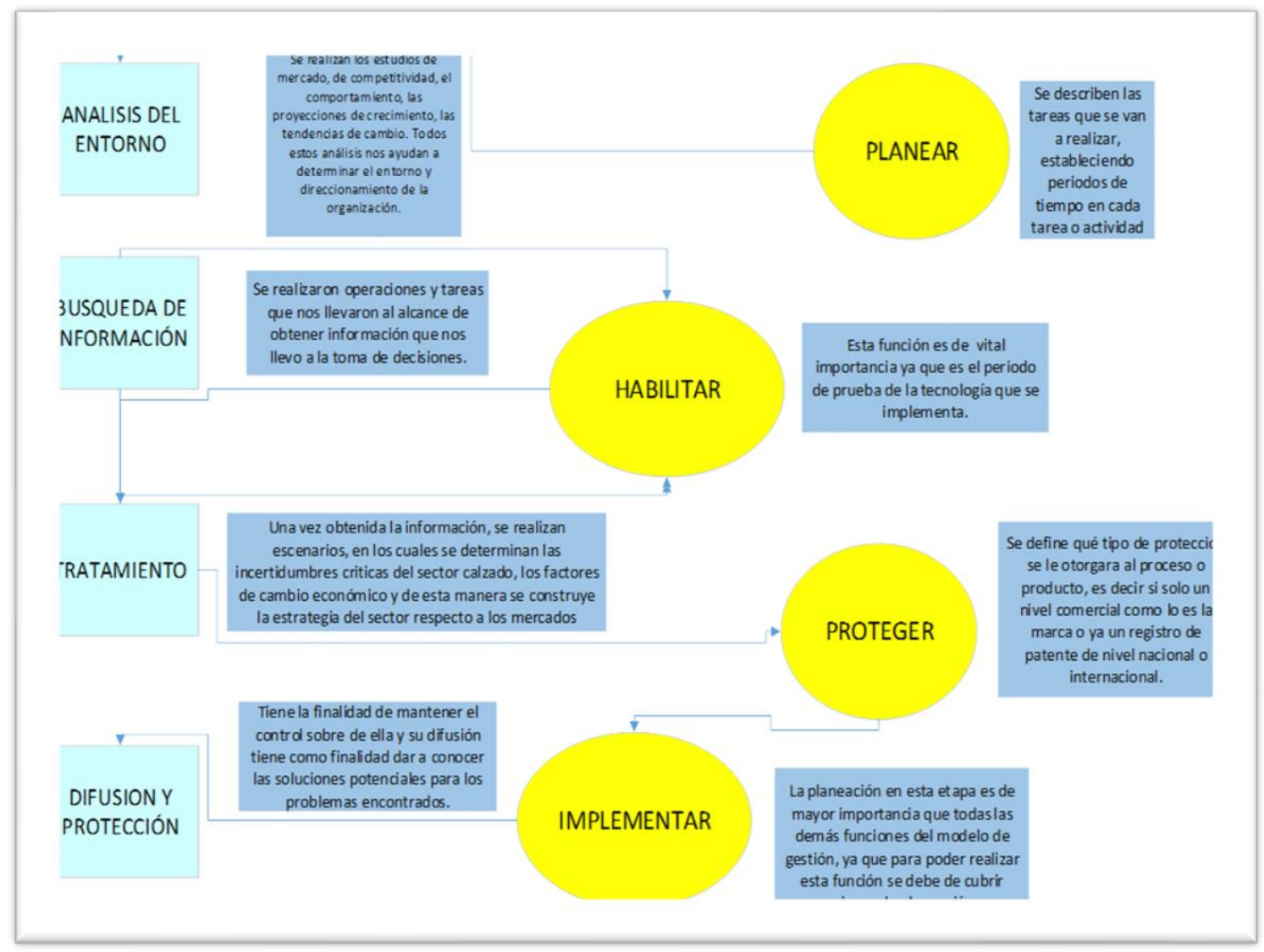

Fuente: Elaboración propia 


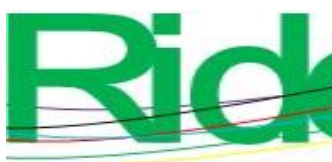

Revista Iberoamericana para la Investigación y el Desarrollo Educativo ISSN $2007-7467$

El principal objetivo del modelo de gestión tecnológica dentro de la organización es ayudar a desarrollar y evolucionar los sistemas, métodos, procesos y tecnologías con el propósito de innovar los existentes, así como ayudar a evaluar la nueva tecnología para tomar la mejor decisión. La innovación surge mediante el modelo nacional de gestión de tecnología debido a que contiene las siguientes funciones: vigilancia tecnológica, planeación básica, implementación y mejora, por lo que no se utilizan las funciones vitales de habilitar y proteger. Por ello, se continúa fortaleciéndolo con base en el modelo que propone el Premio Nacional de Tecnología e Innovación.

Vale acotar que las funciones estratégicas que marca el modelo de gestión tecnológica de la empresa descritas en la figura 8 se explican en seguida, así como el diagnóstico de necesidades de capacitación (DNC) con las competencias identificadas como necesarias a desarrollar por el personal gerencial; asimismo, su nivel requerido técnicamente por cada función y respaldado por la dirección general para la transición e implementación del modelo en cuestión. En este sentido, la descripción de los niveles del diagnóstico de necesidades de capacitación (DNC) para el personal gerencial se presenta a continuación:

- Nivel 1: Competencia no necesaria

- Nivel 2: Competencia baja

- Nivel 3: Competencia intermedia

- Nivel 4: Competencia en dominio alto y profundo

- Nivel 5: Competencia en grado experto y con capacidad para enseñar a otros.

Vigilar: Se realiza una acción continua de monitoreo y búsqueda en el entorno de las principales tecnología y desarrollos tecnológicos que impactan a nuestro negocio, así como tendencias e indicadores sectoriales.

DNC gerencial, competencias identificadas:

- Pensamiento estratégico $\rightarrow 90 \%$ nivel 5

- Vigilancia tecnológica $\rightarrow 96 \%$ nivel 5

- Indagación de tendencias $\rightarrow 95 \%$ nivel 4 y 5

- Investigación $\rightarrow 90 \%$ nivel 4

Planear: Se realizó la planeación tecnológica y estratégica de acuerdo con los resultados obtenidos en la vigilancia tecnológica. Uno de ellos era la apertura del mercado chino en México. 
DNC gerencial, competencias identificadas:

- Pensamiento analítico $\rightarrow 86 \%$ nivel 5

- Capacidad de innovación $\rightarrow 100 \%$ nivel 5

- Desarrollo de propuestas de mejora $\rightarrow 77 \%$ nivel 4

- Gestión de nuevas tecnologías $\rightarrow 95 \%$ nivel 5

- Investigación $\rightarrow>90 \%$ nivel 4

Habilitar: Para la obtención de las tecnologías la empresa realiza la gestión financiera con apoyos gubernamentales — p. ej., el Consejo Nacional de Ciencia y Tecnología (Conacyt), los Fondos Guanajuato y la Secretaría de Economía (SE)—. Para identificar el capital intelectual necesario se elabora un perfil según los requerimientos de adopción y trasferencia de tecnología. Desarrollan con vinculaciones de universidades y centros de investigación; asimismo, tenemos alianzas estratégicas con los mejores fabricantes de tecnología y moda de Italia.

DNC gerencial, competencias identificadas

- Búsqueda de información $\rightarrow 70 \%$ nivel 5

- Innovación del conocimiento $\rightarrow 93 \%$ nivel 5

- Inteligencia emocional $\rightarrow 80 \%$ nivel 4

- Negociación $\rightarrow 95 \%$ nivel 5

Proteger: Se creó la estrategia de registro y protección ante el Instituto Mexicano de Propiedad Industrial (IMPI) para el resguardo de las tecnologías desarrolladas, en trámite actual la solicitud de patente en producto y proceso.

DNC gerencial, competencias identificadas:

- Pensamiento sistémico $\rightarrow 89 \%$ nivel 5

- Trabajo en equipo $\rightarrow 93 \%$ nivel 5

- Liderazgo situacional $\rightarrow 97 \%$ nivel 4

- Proyectos en gestión tecnológica $\rightarrow 90 \%$ nivel 5

- $\quad$ Normatividad aplicable $\rightarrow 90 \%$ nivel 4

- $\quad$ Propiedad industrial $\rightarrow 94 \%$ nivel 5

Implementar: Es la ejecución del plan tecnológico y es el resultado de la habilitación tecnológica para conseguir los objetivos planteados desde el inicio.

DNC gerencial, competencias identificadas:

- Gestión del talento humano $\rightarrow 91 \%$ nivel 5

- Habilidad para comunicar $\rightarrow 89 \%$ nivel 5

- Capacidad para escuchar a otros $\rightarrow 97 \%$ nivel 4

- Iniciativa $\rightarrow 96 \%$ nivel 5 
- $\quad$ Mejora continua $\rightarrow 86 \%$ nivel 4

Los resultados de aplicación práctica de la presente investigación se traducen en los siguientes entregables para la organización:

- Diagnóstico de necesidades de capacitación gerencial y operativa (DNC).

- Plan de capacitación por competencias 2018-2020 para puestos gerenciales.

- Descripciones de puesto gerenciales traducidas a competencias con seguimiento de indicadores de desempeño (KPI's)

- Evaluación piloto para el desempeño gerencial bajo la metodología 360 grados para $100 \%$ de las posiciones. En seguida se presenta el análisis gráfico por posición en la evaluación piloto, y se enseñan los hallazgos relevantes del proceso, con una interpretación sintética de sus principales mediciones.

\section{Gerencia general}

En cuanto a liderazgo, se encuentra un punto debajo del requerido, por lo que se necesita mejorar la comprensión de la organización, delegación y seguimiento de planes, programas y proyectos de los colaboradores (figura 9):

Figura 9: Capacidades de rol gerencial (puesto: gerente general)

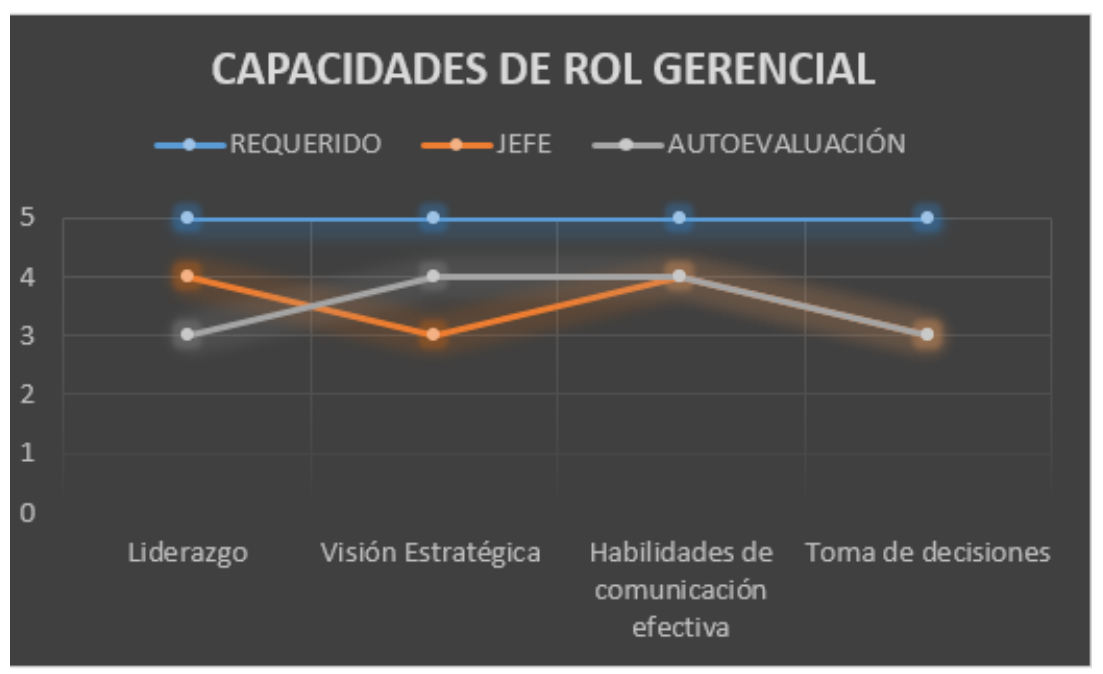

Fuente: Elaboración propia

Esta variable presenta como área de mejora la visión estratégica, lo cual se puede mejorar con la habilidad de comprensión y anticipándose a los cambios del mercado para encontrar oportunidades de negocio. Su comunicación es mayormente efectiva, sin embargo, se necesita entender y proporcionar mayor seguimiento y solución a los problemas internos del personal. 


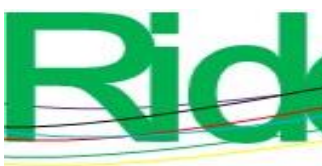

Revista Iberoamericana para la Investigación y el Desarrollo Educativo ISSN $2007-7467$

Por otra parte, presenta una brecha importante en la toma de decisiones, lo cual se debe optimizar, ya que de ello depende la planeación y la mejora de la organización. Asimismo, requiere mayor orientación al servicio, el cual emerge de la visión estratégica, por lo que es un área de oportunidad importante para la gerencia general. Esta se caracteriza por tener un nivel adecuado de negociación, aspecto primordial en el liderazgo de su función. Requiere elevar su nivel de inteligencia emocional, así como generar más proyectos de gestión de talento para fortalecer el liderazgo.

Sobre el modelo de gestión tecnológica, las competencias que debe mejorar el gerente general son negociación, inteligencia emocional y proyectos en la gestión del talento (figura 10):

Figura 10. Genéricas por el modelo de gestión tecnológica

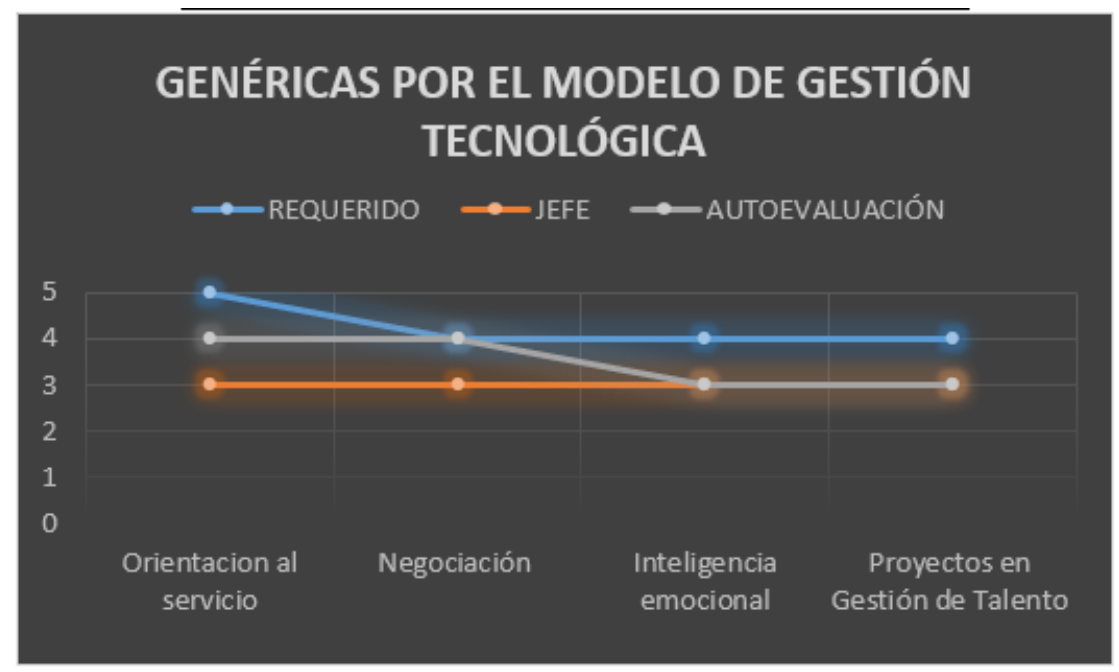

Fuente: Elaboración propia

\section{Gerencia de recursos humanos}

Este aspecto presenta una brecha importante del desarrollo de las personas entre el nivel de competencia y el nivel requerido. Por tanto, se debe mejorar la orientación para conseguir óptimos resultados (figura 11). 


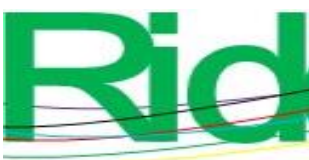

Revista Iberoamericana para la Investigación y el Desarrollo Educativo ISSN $2007-7467$

Figura 11. Capacidades de rol gerencial (puesto: gerente de recursos humanos)

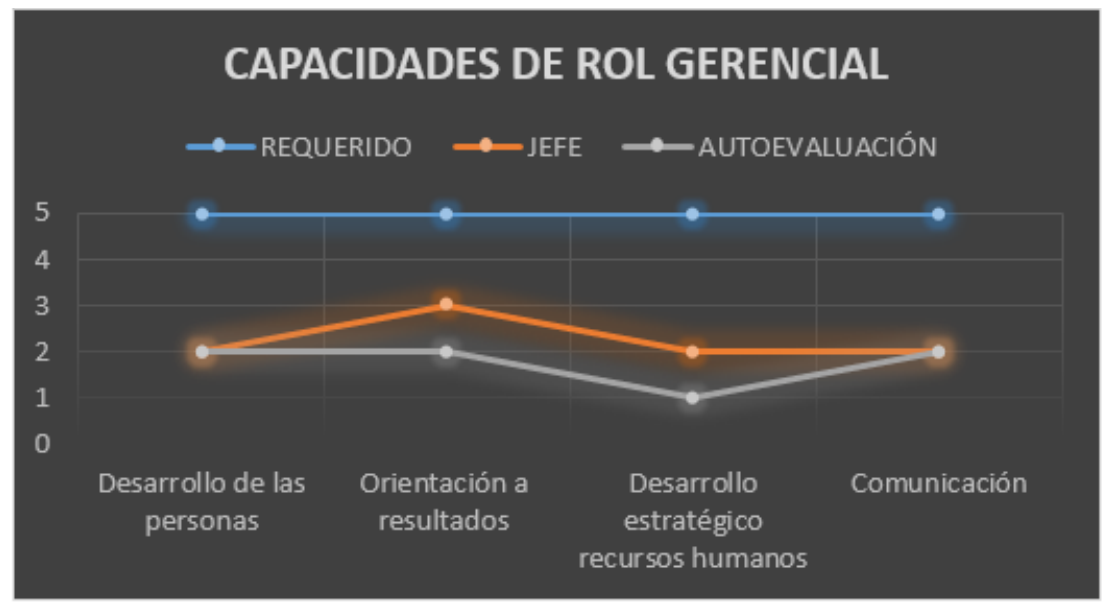

Fuente: Elaboración propia

En cuanto al desarrollo estratégico de recursos humanos, se deben implementar estrategias para aprovechar el talento de los colaboradores. En lo concerniente a comunicación, su nivel se halla por debajo de lo requerido para su puesto, lo que es primordial para el estrechamiento de relaciones interpersonales.

Asimismo, se debe trabajar en una mayor orientación al servicio, lo cual emerge de la visión estratégica, de ahí que se considere un área de oportunidad importante para su función como gerente de recursos humanos. Del mismo modo, muestra una brecha importante en la competencia de la negociación, lo que es crítico para desempeñar las responsabilidades como mediador entre la organización y los colaboradores. También se debe hacer énfasis en el nivel de inteligencia emocional para fortalecer las habilidades de comunicación y desarrollo de personas (figura 12).

Figura 12. Genéricas por el modelo de gestión tecnológica

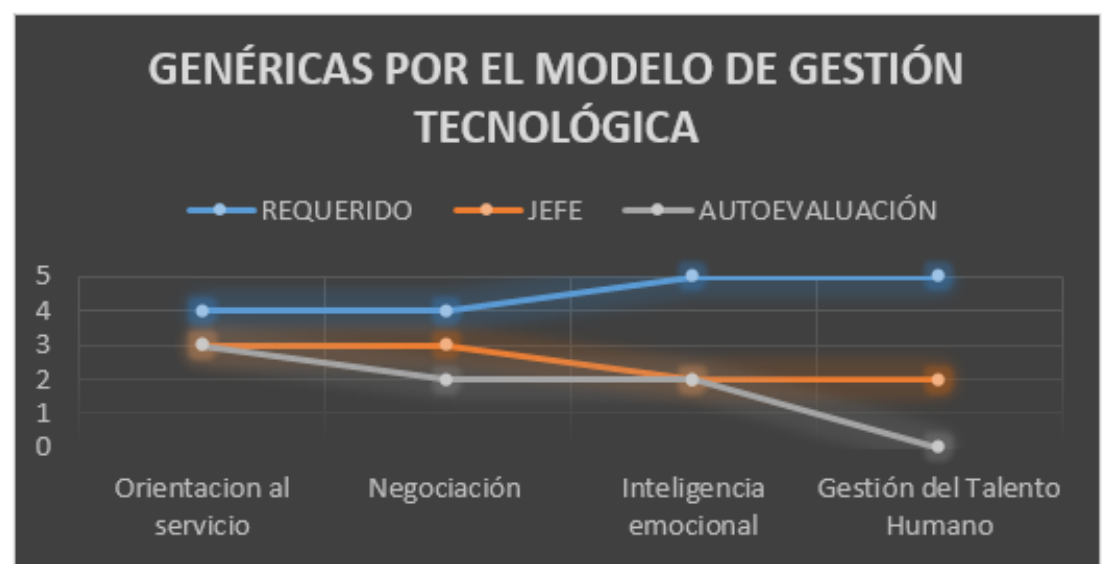

Fuente: Elaboración propia 


\section{Gerencia de contabilidad}

El liderazgo se halla un punto debajo del requerido, por lo que necesita mejorar la comprensión de la organización, delegación y seguimiento de planes, programas y proyectos de los colaboradores (figura 13). Asimismo, se debe optimizar la orientación hacia los resultados, pues se tiende a olvidar su enfoque, lo que pone en riesgo el logro de los objetivos trazados.

Figura 13. Capacidades del rol gerencial (puesto: gerente de contabilidad)

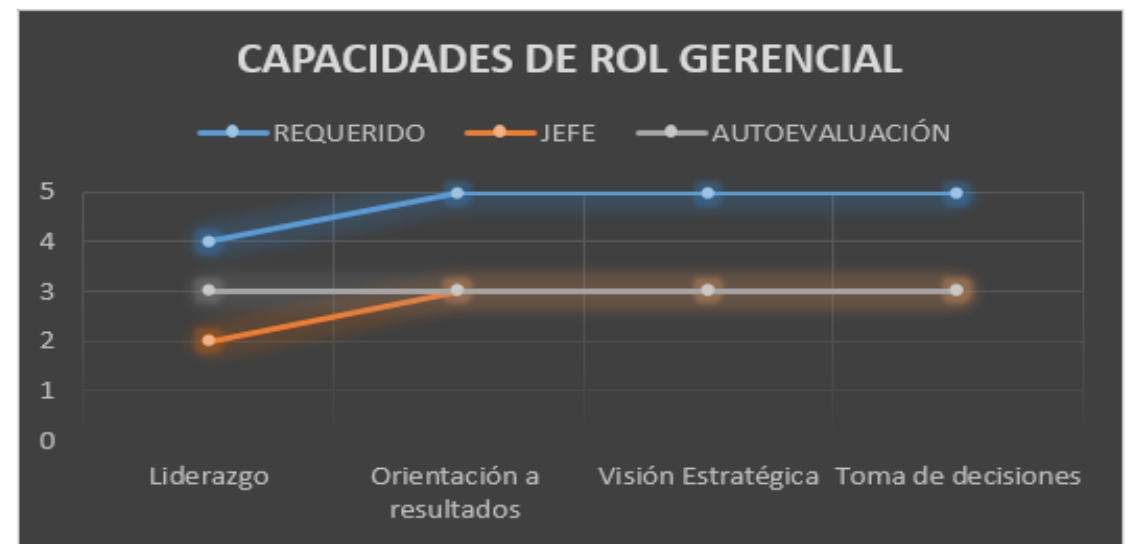

Fuente: Elaboración propia

Como área de mejora se detectó la visión estratégica, lo que se puede atender con la habilidad de comprensión y anticipándose a los cambios del mercado para encontrar oportunidades de negocio. También se evidencia una brecha importante en la toma de decisiones, la cual depende de la planeación y la mejora de los procesos de su área.

Igualmente, se requiere mayor orientación al servicio, la cual emerge de la visión estratégica. En tal sentido, se debe tener un nivel adecuado de negociación, aspecto primordial para la ejecución de su labor, ya que se relaciona directamente con proveedores y clientes internos y externos. Se sugiere, por ende, elevar el nivel de inteligencia emocional, la cual se halla por debajo de lo recomendado.

Sobre la capacidad de innovación, se necesita mayor adaptación al cambio, crear nuevas estrategias y dar un mejor acompañamiento a su implementación. Es necesario que se mejore el desarrollo de nuevas propuestas, ya que esta variable se halla por debajo de la competencia requerida para la transición del modelo. Asimismo, en la gestión de nuevas tecnologías se deben implementar estrategias para optimizar el aprovechamiento de su departamento (figura 14). 
Figura 14. Genéricas del modelo de gestión tecnológica

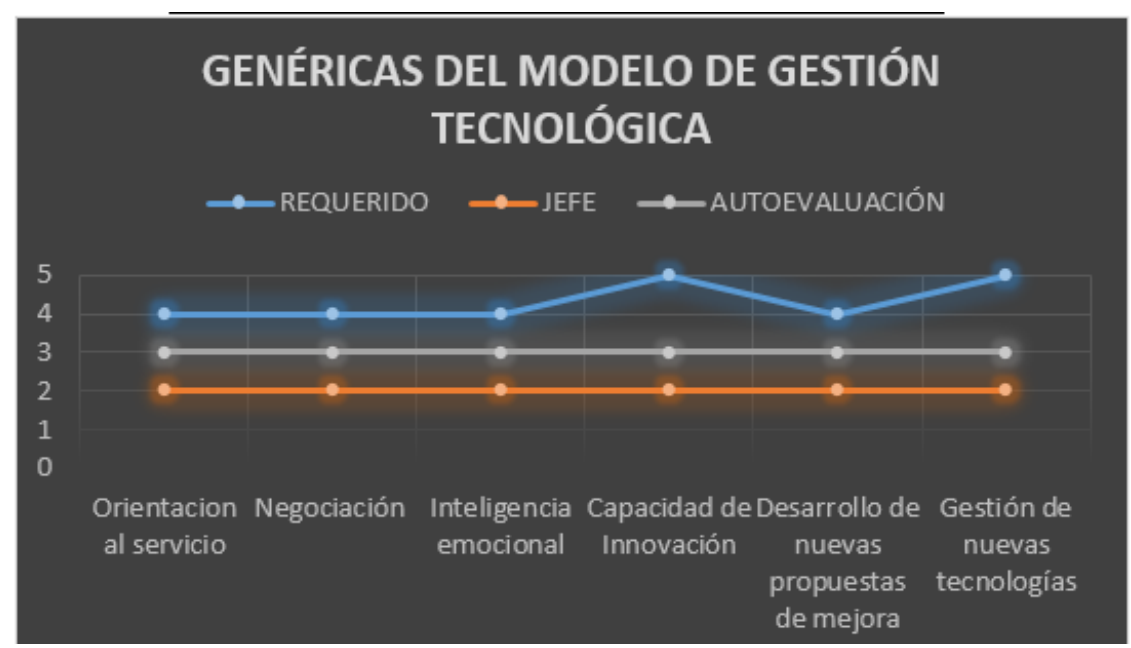

Fuente: Elaboración propia

\section{Gerencia de ventas}

Esta categoría presenta un nivel adecuado de liderazgo, aunque se puede mejorar para alcanzar el requerido. Asimismo, se debe trabajar en la orientación a resultados para no perder de vista los objetivos trazados. El área de oportunidad radica en las habilidades de comunicación efectiva, por lo que se debe potenciar el entendimiento y proporcionar un mayor seguimiento, así como la solución a los problemas internos del personal. También se debe hacer énfasis en la toma de decisiones, ya que de esta depende la planeación y la mejora de la organización (figura 15).

Figura 15. Capacidades de rol gerencial (puesto: gerente de ventas)

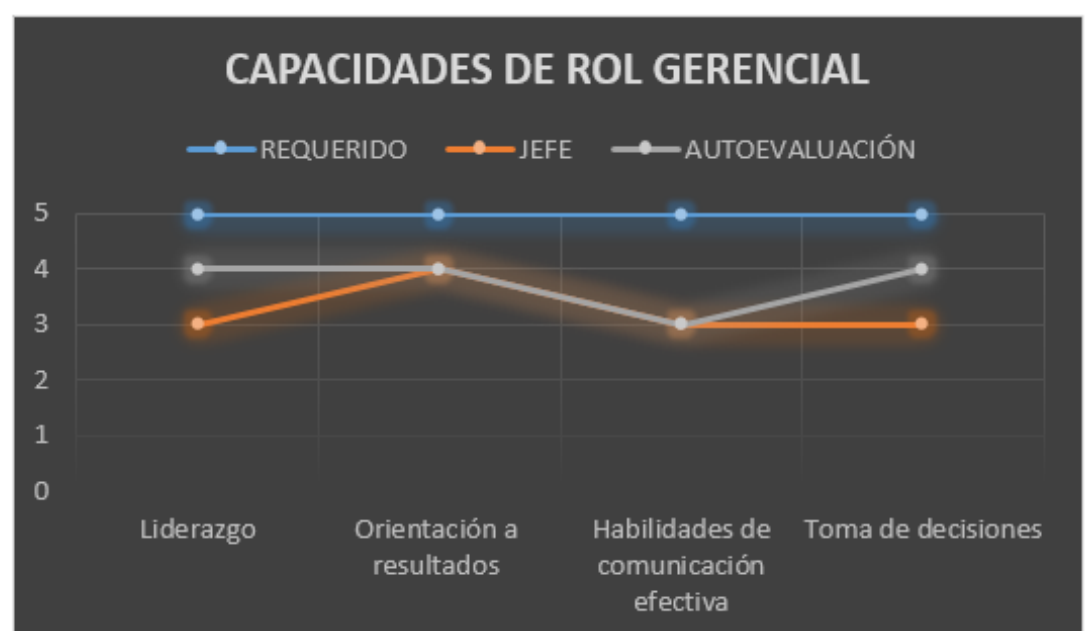

Fuente: Elaboración propia

Igualmente, se requiere mayor orientación al servicio, el cual emerge de la visión estratégica, por lo que es un área de oportunidad importante para la gerencia de 
contabilidad, lo que permitirá generar nuevas estrategias y dar un mejor acompañamiento a su implementación.

Es deseable, además, que se optimice el desarrollo de nuevas propuestas de mejora, ya que estas se encuentran por debajo de la competencia requerida para la transición del modelo. En pocas palabras, el diagnóstico realizado demuestra que se deben implementar estrategias para aprovechar las tareas desempeñadas por este departamento.

Figura 16. Genéricas por el modelo de gestión tecnológica

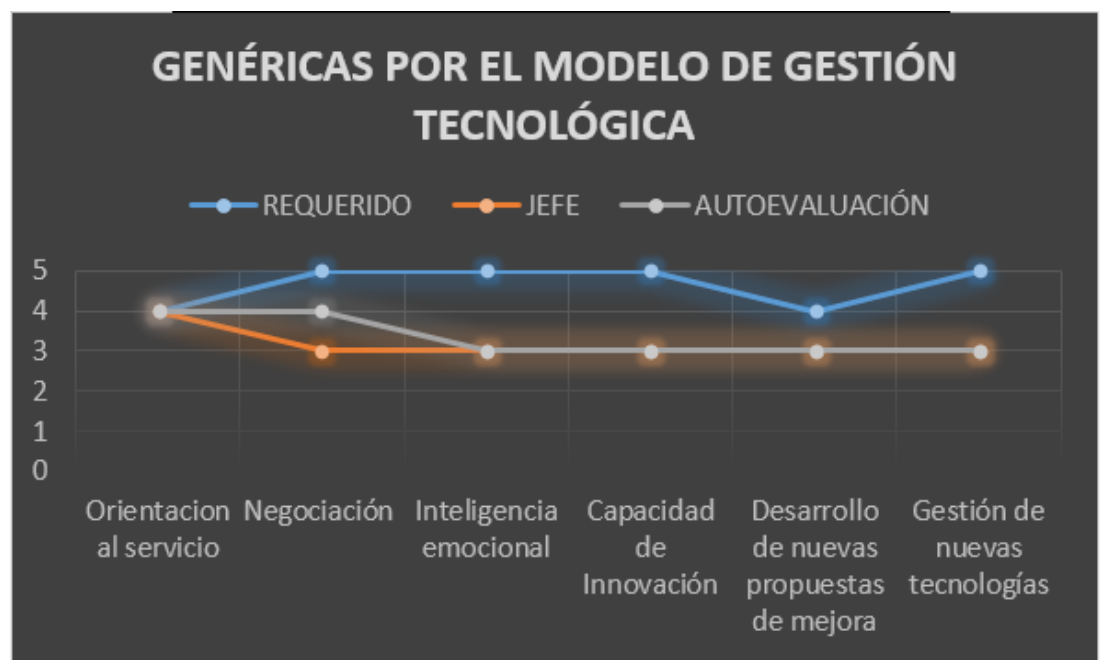

Fuente: Elaboración propia

\section{Gerencia de calidad}

Se debe mejorar la orientación a los resultados. Si bien la calidad en el trabajo cuenta con un nivel adecuado, se debe elevar esta competencia al menos en un nivel adicional (figura 17). Es deseable que se trabaje en aumentar su adaptabilidad para el cambio, ya que sus procesos están expuestos a cambios constantes para cumplir con las metas establecidas. Por otra parte, se detectaron áreas de oportunidad en cuanto al liderazgo para el cambio y la adaptabilidad para el cambio. 
Figura 17. Capacidades de rol gerencial (puesto: gerente de calidad)

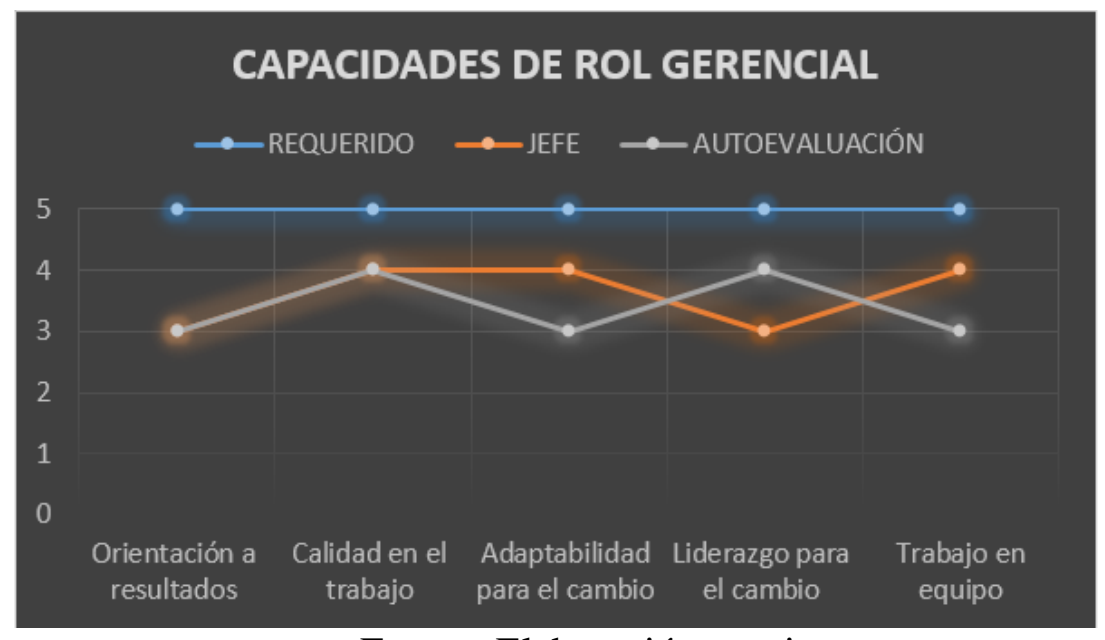

Fuente: Elaboración propia

Se requiere enfocarse en el trabajo en equipo para alcanzar metas y apoyar la transición de la organización. Asimismo, se debe elevar el nivel de inteligencia emocional, ya que este se halla por debajo del requerido.

Figura 18. Genéricas por el modelo de gestión tecnológica

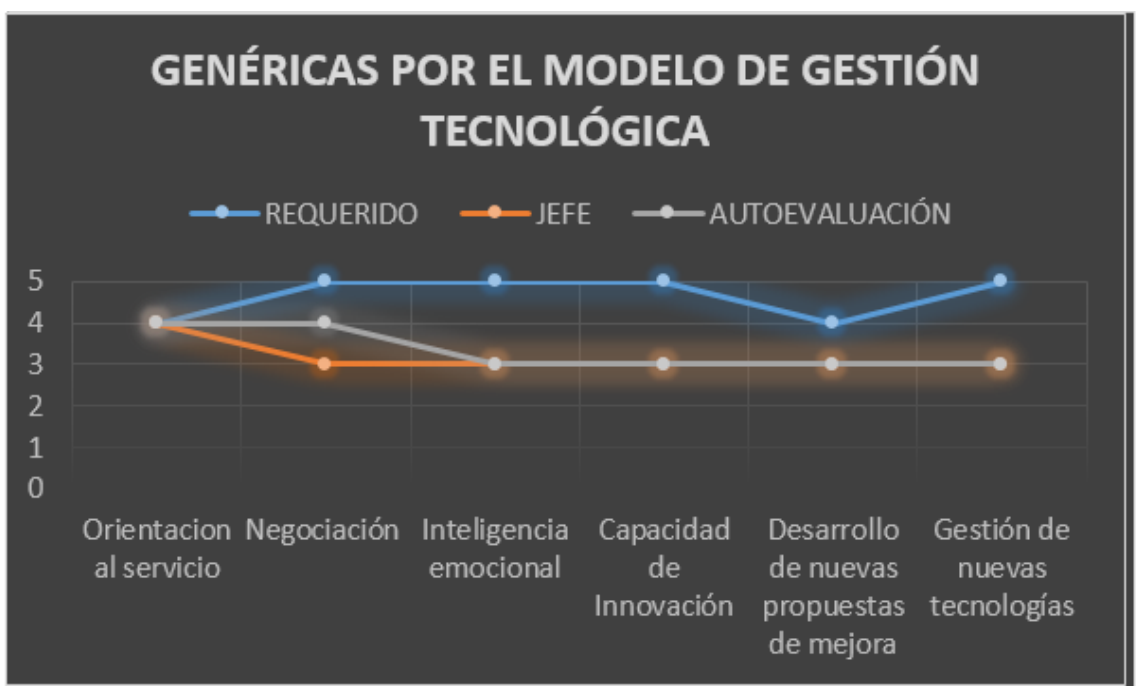

Fuente: Elaboración propia

En cuanto a productividad, tiene un nivel adecuado, aunque puede alcanzar indicadores más altos. Presenta una brecha importante del desarrollo de las personas entre el nivel de competencia que tiene y el nivel requerido. Además, se debe concientizar a las personas sobre la responsabilidad que se tiene en el área. En el sentido de capacidad de innovación, se necesita una mayor adaptabilidad al cambio, crear nuevas estrategias y dar un mejor acompañamiento a su implementación (figura 18). 


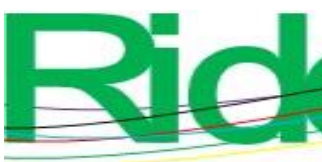

Revista Iberoamericana para la Investigación y el Desarrollo Educativo ISSN $2007-7467$

Es necesario potenciar también el desarrollo de nuevas propuestas de mejora, ya que se encuentra por debajo de la competencia requerida para la transición del modelo. En cuanto a innovación para el conocimiento, resulta deseable ampliar la visión con la implementación de estrategias para adquirir más información técnica del área (figura 18).

\section{Gerencia de mantenimiento}

El nivel de liderazgo se encuentra muy por debajo del requerido, por lo que necesita mejorar la comprensión de la organización, delegación y seguimiento de planes, programas y proyectos de los colaboradores, pues de esto depende la toma de decisiones (figura 19).

Figura 19. Capacidades de rol gerencial (puesto: gerente de mantenimiento)

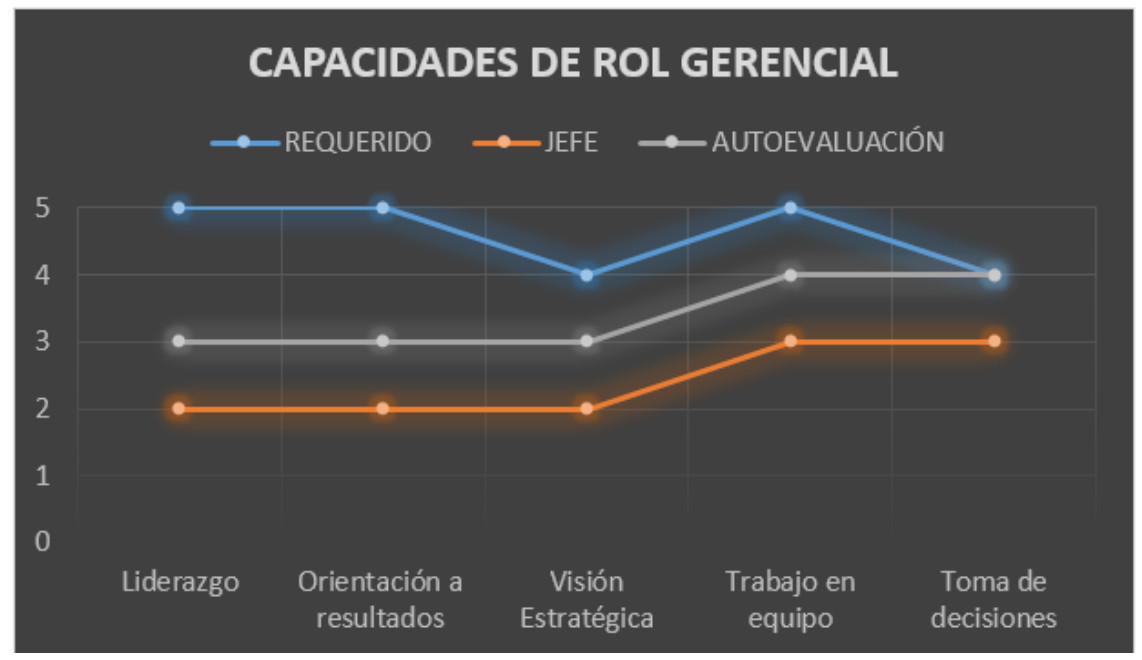

Fuente: Elaboración propia

Asimismo, no se debe perder de vista el objetivo gerencial, ya que esto pone en riesgo el logro de las metas planteadas (figura 19). En cuanto a una área de mejora, se debe tener en cuenta la visión estratégica, lo que se puede atender con la habilidad de comprensión y anticipándose a los cambios del mercado para encontrar oportunidades de negocio. Es indispensable que se haga énfasis también en la habilidad de trabajo en equipo para que se puedan lograr metas generales y apoyar la transición de la organización. Respecto a la evaluación de competencias que respalden el modelo de gestión tecnológica, el gerente de mantenimiento un nivel 3 en cuanto a negociación y capacidad de innovación, lo que coincide con el requerimiento del perfil del puesto y la evaluación del jefe inmediato. No obstante, se puede constatar en la figura 20 las competencias de orientación a resultados, gestión de nuevas tecnologías e inteligencia emocional como 
oportunidades para elevar la competencia profesional al menos en un nivel de mejora latente.

Figura 20. Genéricas por el modelo de gestión tecnológica

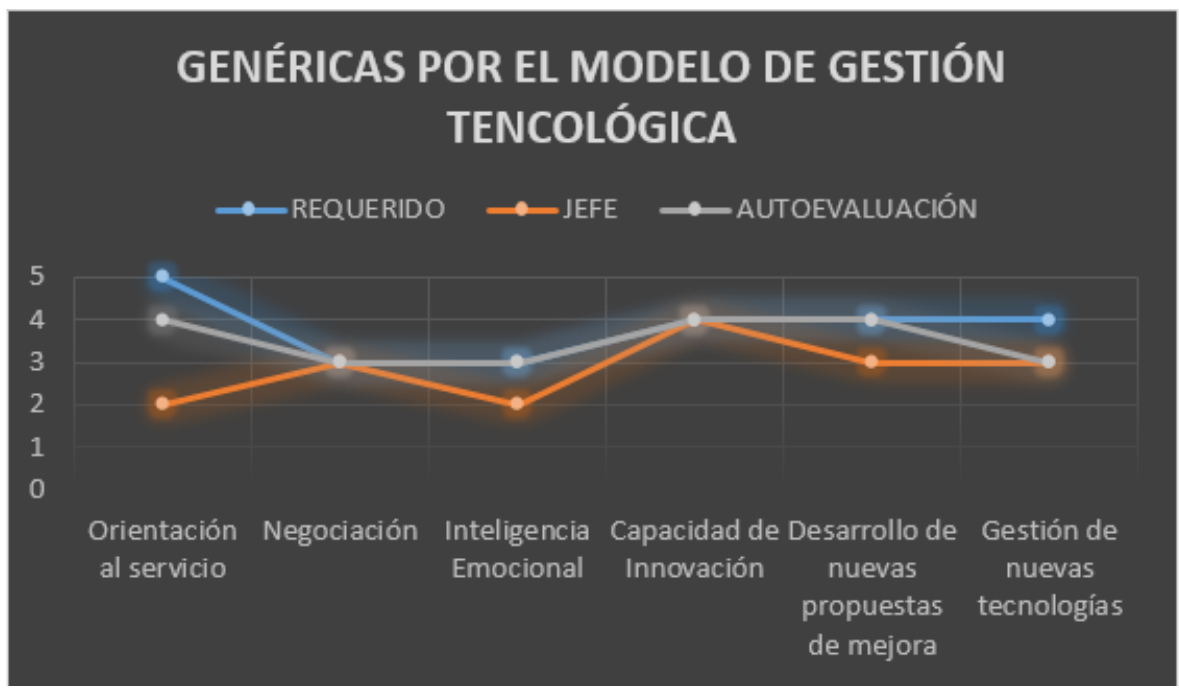

Fuente: Elaboración propia

\section{Gerencia de sistemas}

Para el análisis de esta gerencia, los resultados se muestran en la figura 21. En liderazgo se observa una evaluación del jefe inmediato en nivel 4 , lo que coincide con su autoevaluación; no obstante, el perfil requerido es nivel 5, por lo que se puede decir que existe un área de mejora.

Figura 21. Capacidades de rol gerencial (puesto: gerente de sistemas)

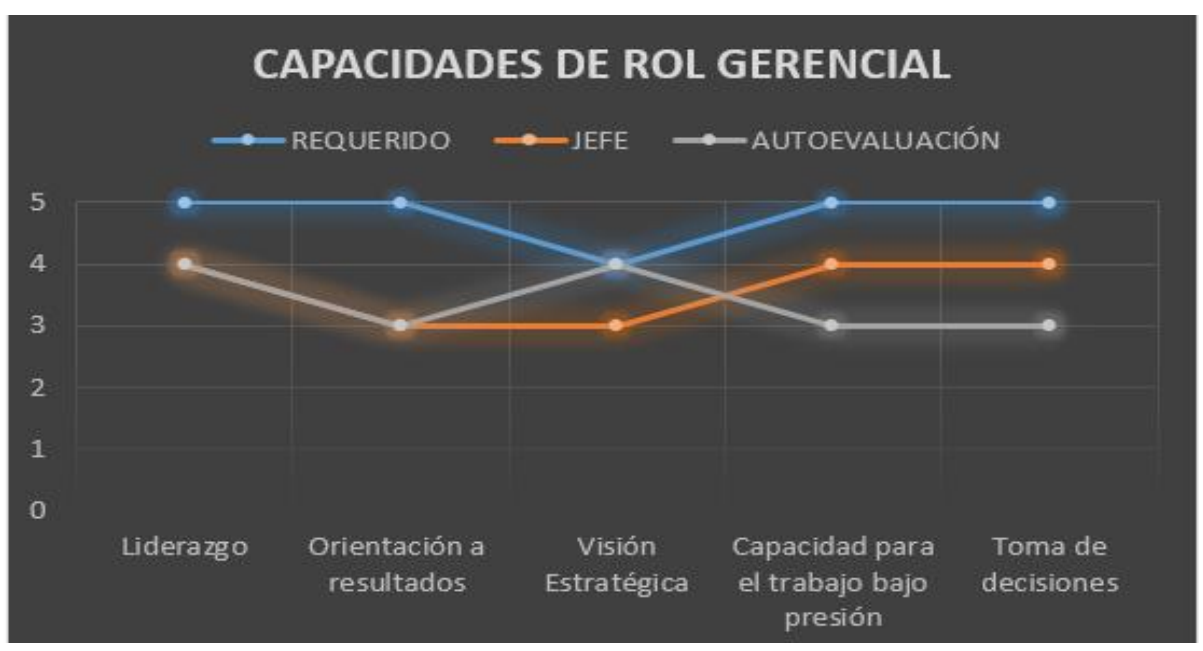

Fuente: Elaboración propia 


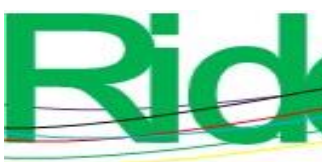

Revista Iberoamericana para la Investigación y el Desarrollo Educativo ISSN 2007 - 7467

Asimismo, en orientación a resultados, capacidad para el trabajo bajo presión y toma de decisiones se detectó un nivel inferior al requerido por el perfil; aunque vale acotar que en visión estratégica se alcanzó un nivel 4 de competencia técnica necesaria (figura 21).

En cuanto a la evaluación de sus capacidades de acuerdo al DNC para respaldar el modelo de gestión tecnológica, es necesario para el gerente de sistemas trabajar en la inteligencia emocional. Para el desarrollo gerencial se deben tener en cuenta capacidades como la orientación al servicio y la negociación, las cuales se presentan en nivel 4 y 3 , respectivamente.

Figura 22. Genéricas por el modelo de gestión tecnológica

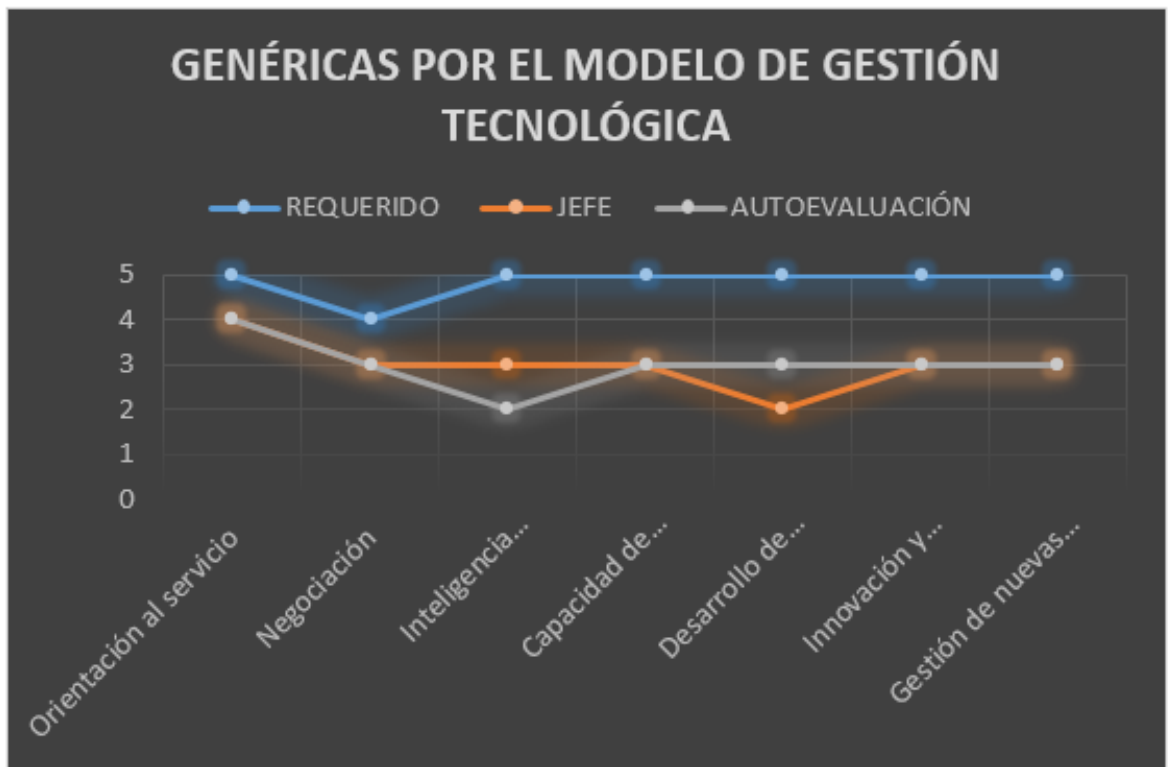

Fuente: Elaboración propia

\section{Gerencia de proyectos}

Para esta gerencia en la figura 23 se observa que la autoevaluación del ocupante del puesto se halla en el nivel 3, con la evaluación de su jefe inmediato para la capacidad de liderazgo. No obstante, la autoevaluación en orientación de resultados y visión estratégica se sitúan en un nivel 4, a pesar de que el perfil demanda un nivel 5 en todas las competencias. Se recomienda, por tanto, potenciar su capacidad de visión estratégica y la toma de decisiones en al menos 2 niveles para reconfigurar su liderazgo y la orientación de resultados. 


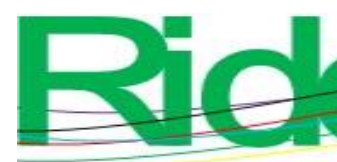

Revista lberoamericana para la Investigación y el Desarrollo Educativo ISSN 2007 - 7467

Figura 23. Capacidades de rol gerencial (puesto: gerente de proyectos)

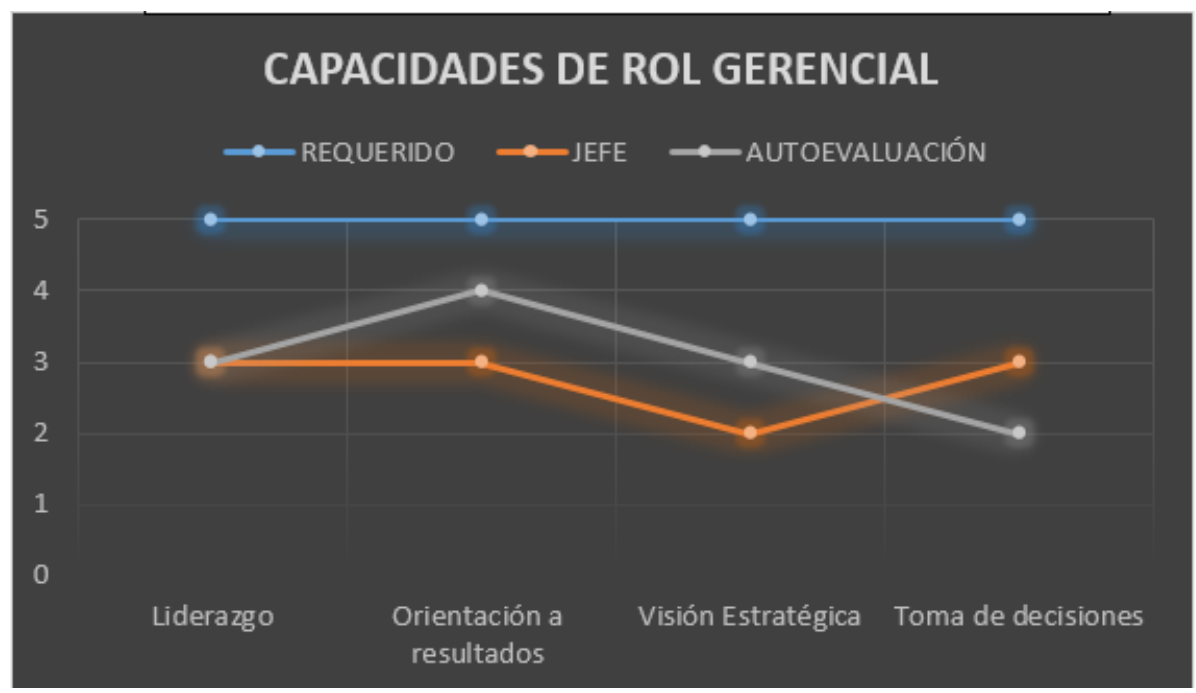

Fuente: Elaboración propia

Por último, la capacidad de inteligencia emocional e innovación se debe desarrollar no solo de forma individual, sino colaborativa, ya que este gerente mantiene relación con los demás para seguir los proyectos tecnológicos en cada una de las áreas (figura 24).

Figura 24. Genéricas por el modelo de gestión tecnológica

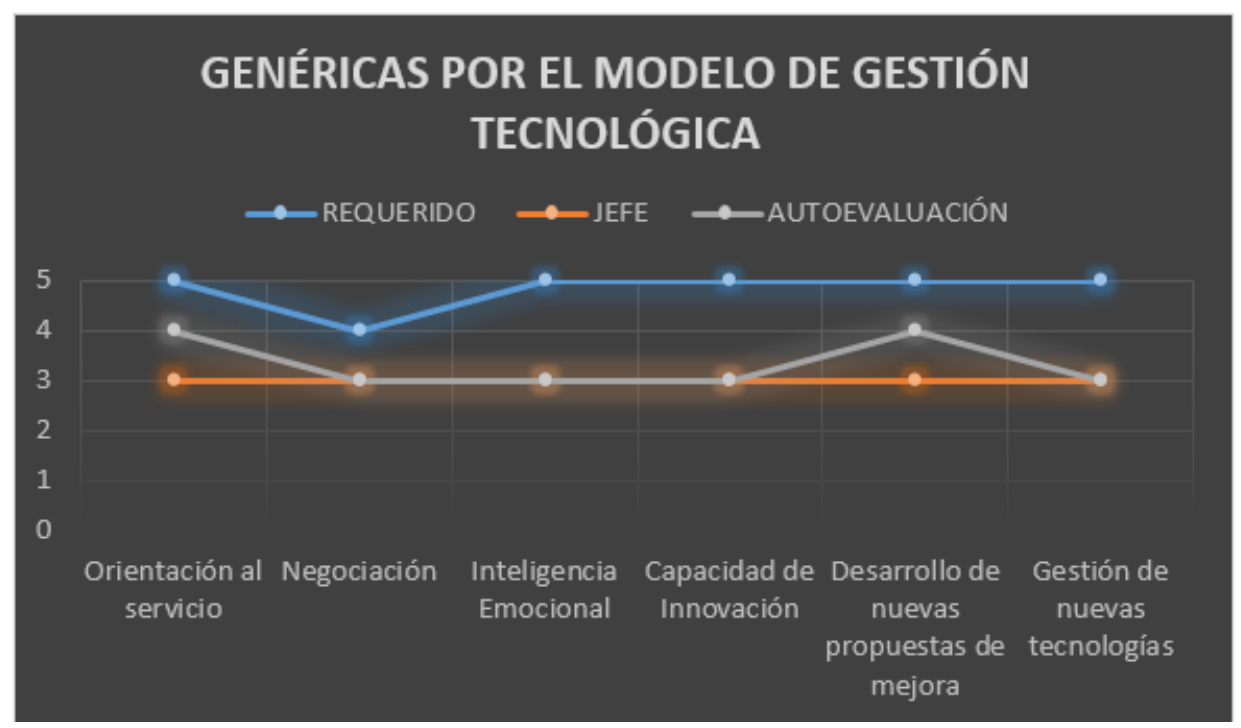

Fuente: Elaboración propia 


\section{Discusión}

Los resultados recabados con la presente investigación permiten indicar como fortaleza medular la gestión del conocimiento de la propia organización, especialmente en cuanto al desarrollo de competencias (profesionales genéricas) y comportamentales (del rol gerencial) exigidas en un perfil nivel 5 (competencia en grado experto y con capacidad para enseñar a otros).

Por otra parte, en las evaluaciones efectuadas al desempeño gerencial se aprecian conocimientos, habilidades y actitudes de los ocupantes de puestos gerenciales en nivel 3 (competencia intermedia) y nivel 4 (competencia en dominio alto y profundo respectivamente). En este sentido, es notable la diferencia o breca existente entre la competencia actual gerencial y la exigida por la dirección de la empresa para respaldar y adoptar su modelo de gestión tecnológica traducido en el día a día de sus colaboradores.

Sobre esta línea de análisis, se debe tomar en cuenta que el ámbito de desarrollo de competencias profesionales es un proceso de transición, que depende de desempeños integrales para identificar, interpretar, argumentar y resolver problemas del contexto, con idoneidad, compromiso ético y mejoramiento continuo, integrando sistémicamente el saber ser, el saber convivir, el saber hacer y el saber conocer (Tobón, 2012).

Asimismo, la segunda fortaleza relevante que aporta la investigación es la socialización del conocimiento tácito y explícito de los gerentes y sus propios equipos de trabajo, de forma individual con el logro consciente del alcance de responsabilidad y su afectación positiva y/o negativa en el trabajo de los demás. Por otra parte, y en forma colectiva, logran la certeza del funcionamiento de sus equipos de trabajo como un todo integrado, es decir, de manera sistémica son capaces de trabajar en talleres, sesiones de diseño de descriptivos de sus puestos e indicadores de evaluación por puesto gerencial con el objetivo compartido de identificar, construir, documentar y evaluar sus desempeños profesionales con impacto en su formación integral, mejora de sus resultados y compensación de ellos.

En cuanto a las limitaciones del estudio, se pueden señalar el diagnóstico de necesidades de capacitación (DNC), así como la evaluación al desempeño 360 grados realizada por el diagnóstico situacional, pues se deben efectuar de forma integral para todas las posiciones de trabajo y como estrategia sistemática de mejora continua. En este sentido, consideramos que de ello dependerá la adopción y transición hacia su modelo de gestión tecnológica de la organización. 


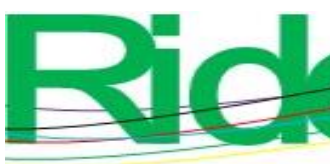

Revista Iberoamericana para la Investigación y el Desarrollo Educativo ISSN $2007-7467$

Otra limitación tiene que ver con la continuación del monitoreo y la evaluación 360 grados de forma completa, incluyendo dentro del perfil de puesto competencias blandas (actitudinales), genéricas (para apoyar el modelo de gestión tecnológica) y técnicas específicas (alcance de resultados) para detectar probables áreas de productividad, innovación y transferencia tecnológica. Por ello, se sugiere el acompañamiento y retroalimentación oportuna, así como su dispersión de resultados por área y puesto específico para la toma directiva de decisiones.

La aplicación del método de evaluación del desempeño 360 grados es reproducible y repetible a partir de la detección de necesidades de capacitación (DNC) en forma objetiva y sistemática. Este proceso llevado a cabo durante la investigación sirvió para detectar las competencias, habilidades y actitudes que se pueden considerar como áreas de mejora para lograr los objetivos organizacionales de la empresa durante su transición al modelo de gestión tecnológica creado para la competitividad global.

La investigación, además, aportó una herramienta significativa para dar seguimiento al diagnóstico de necesidades de capacitación (DNC), las cuales fueron atendidas en la actualización de las descripciones de puestos por competencias (descripción del empleo) para finalmente ser evaluadas a partir de la evaluación del desempeño (metodología 360 grados) en cuanto al saber (conocimiento), saber ser (actitudes) y saber hacer (habilidades) gerenciales actuales y potenciales a desarrollar en los colaboradores de forma integral.

En síntesis, se sugiere realizar otras evaluaciones sobre el desempeño como proceso sistemático de mejora continua para la organización. Para ello, es necesario diagnosticar las competencias técnico-específicas en puestos gerenciales y operativos en futuras intervenciones organizacionales, a partir de las descripciones de puesto logradas en el presente estudio, documento de análisis base para el registro de los ajustes potenciales en competencias. Cabe destacar que la evaluación del desempeño 360 grados es susceptible de aplicación en lo sucesivo para efectos de medir el avance de las herramientas de detección, diagnóstico, tratamiento de información y evaluación al desempeño con base en competencias profesionales. 


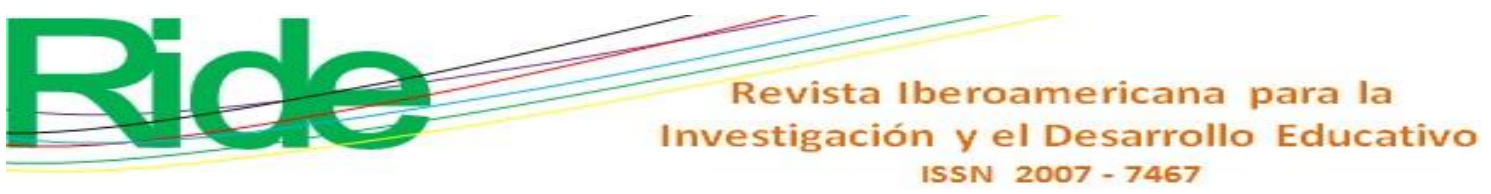

\section{Conclusiones}

En cuanto al proceso de evaluación, este se debe centrar en las potencialidades personales y profesionales de los colaboradores y gerentes involucrados para detectar las oportunidades de mejora consistentes en la adopción de nuevos conocimientos y la comprensión del impacto de sus saberes actuales. Esto permitiría a las personas su aplicación en un contexto laboral y personal determinado, así como la adopción de herramientas técnicas y prácticas para el desarrollo de su función.

La evaluación es pertinente y éticamente viable para ser desarrollada de forma imparcial con un enfoque en las potencialidades a partir de un diagnóstico situacional preciso. No obstante, también se pueden usar otras metodologías alternas para enriquecer los ambientes en los cuales se desempeña la persona. Esto significa que dentro de la organización pueden existir fuerzas impulsoras para desarrollar las competencias identificadas superando las brechas individuales sujetas a medición continua y con trazabilidad, teniendo en cuenta sus potencialidades reales en forma individual y dentro de sus equipos de trabajo.

Finalmente, en relación con el efecto positivo del desempeño gerencial, se puede decir que los resultados fueron mixtos en cuanto a la evaluación de los puestos en jefaturas, coordinadores y puestos operativos que dependen directamente de ellos. En pocas palabras, se observó una relación positiva en la fijación de objetivos compartidos, redefinición de procesos de trabajo y readaptación de los estilos de liderazgo enfocados al desarrollo de propuestas participativas en los equipos de trabajo en ambos sentidos: hacia la alta dirección como hacia los puestos subordinados a las decisiones gerenciales. Por tanto, se recomienda una evaluación integral para todas las posiciones involucradas por áreas funcionales de la empresa. 


\section{Referencias}

Aponte, L. C., y De la Garza, C. (2007). Planeación y evaluación basadas en competencias. México: Trillas.

Barrios, D. Á. y Domínguez, D. (2019). Innovación y su correlación con el desarrollo económico de México: factores de detonación e impulso. Cadenas de Valor e Innovación, 106.

Etzkowitz, H. (2002). La triple hélice: universidad, industria y gobierno. Implicaciones para las políticas y la evaluación. Estocolmo, Recuperado en: http://www. sivu. edu. mx/portal/noticias/2009/VinculacionLatriplehelice. pdf.

Federal, M. G. (2007). Instituto Nacional de Estadística Geografía e InformáticaINEGI-Mexico.

Hitt, M., Black, H y Porter, L. (2006). Administración. México: Editorial Pearson Prentice Hall.

Muiña, F. E. G., \& López, J. E. N. (2007). Las capacidades tecnológicas y los resultados empresariales: Un estudio empírico en el sector biotecnológico español. Cuadernos de Economía y Dirección de la Empresa, (32), 177-210.

Müller, G. (1995). El caleidoscopio de la competitividad. Revista de la CEPAL.

Palomo, M. Á. y Pedroza, Á. R. (2018). La competitividad empresarial: el desarrollo tecnológico. México: Editorial UANL.

Sapien-Aguilar, A. L., Gutiérrez Diez, M. D. C., Piñón Howlet, L. C. y Araiza Zapata, P. A. (2016). Evaluación del desempeño por el método de 360 grados y por factores de una empresa mexicana. Revista Publicaciones, 46, 13-29. Recuperado

de https://revistaseug.ugr.es/index.php/publicaciones/article/viewFile/5794/526 1

Solleiro, J. L., \& Castañón, R. (2005). Competitividad y sistemas de innovación: los retos para la inserción de México en el contexto global. Revista Iberoamericana, 5(15), 165-197.

Tobón, S. (2012). El enfoque socioformativo y las competencias: ejes claves para transformar la educación. En Tobón, S. y Jaik, A. (coords.), Experiencias de aplicación de las competencias en la educación y el mundo organizacional (pp. 3-31). México: Red Durango de Investigadores Educativos A.C. 
Valle Arias, A., Sánchez Rodríguez, S. M., Núñez Pérez, J. C., González Cabanach, R., González García, J. A., \& Rosário, P. J. S. L. D. F. (2010). Motivación y aprendizaje autorregulado. Interamerican Journal of Psychology.

Verdugo, A. (2017) Casos de éxito. Fundación Premio Nacional de Tecnología e Innovación (PNTi). Modelo Innovación Tecnológica, 1(1), 41-46. Recuperado de https://pnt.org.mx/2019/03/05/horma-xvii/

\begin{tabular}{|c|c|}
\hline Contribución & Autor (es) \\
\hline Conceptualización & Adriana Fragoso Mora. \\
\hline Metodología & $\begin{array}{l}\text { Adriana Fragoso Mora, principal; Laura } \\
\text { Marisela Martínez Sánchez, igual. }\end{array}$ \\
\hline Software & $\begin{array}{l}\text { Adriana Fragoso Mora, principal, igual; } \\
\text { Laura Marisela Martínez Sánchez, igual; } \\
\text { Alba Edith Elías Chávez, igual. }\end{array}$ \\
\hline Validación & $\begin{array}{l}\text { Adriana Fragoso Mora, principal; Laura } \\
\text { Marisela Martínez Sánchez, igual. }\end{array}$ \\
\hline Análisis Formal & $\begin{array}{l}\text { Adriana Fragoso Mora, principal; Laura } \\
\text { Marisela Martínez Sánchez, igual. }\end{array}$ \\
\hline Investigación & $\begin{array}{l}\text { Adriana Fragoso Mora, principal, Laura } \\
\text { Marisela Martínez Sánchez, igual; Alba } \\
\text { Edith Elías Chávez, igual. }\end{array}$ \\
\hline Recursos & $\begin{array}{l}\text { Adriana Fragoso Mora, principal, Laura } \\
\text { Marisela Martínez Sánchez, igual; Alba } \\
\text { Edith Elías Chávez, igual }\end{array}$ \\
\hline Curación de datos & $\begin{array}{l}\text { Adriana Fragoso Mora, principal, Laura } \\
\text { Marisela Martínez Sánchez, igual; Alba } \\
\text { Edith Elías Chávez, igual }\end{array}$ \\
\hline $\begin{array}{l}\text { Escritura-Preparación del borrador } \\
\text { original }\end{array}$ & Adriana Fragoso Mora. \\
\hline Escritura- Revisión y Edición & $\begin{array}{l}\text { Adriana Fragoso Mora, principal, Laura } \\
\text { Marisela Martínez Sánchez, igual; Alba } \\
\text { Edith Elías Chávez, igual }\end{array}$ \\
\hline Visualización & Adriana Fragoso Mora. \\
\hline Administración de Proyectos & Adriana Fragoso Mora. \\
\hline Adquisición de Fondos & Adriana Fragoso Mora. \\
\hline
\end{tabular}

